
cultures

Les cahiers de l'Acedle

6-1 | 2009

L'Alsace au cœur du plurilinguisme (2)

\title{
La photographie, déclencheur d'interaction en classe de langue : négociations entre apprenants
}

\section{Catherine Muller}

\section{OpenEdition}

Journals

Édition électronique

URL : http://journals.openedition.org/rdlc/2172

DOI : $10.4000 /$ rdlc. 2172

ISSN : 1958-5772

Éditeur

ACEDLE

Référence électronique

Catherine Muller, « La photographie, déclencheur d'interaction en classe de langue : négociations entre apprenants », Recherches en didactique des langues et des cultures [En ligne], 6-1 | 2009, mis en ligne le 01 avril 2009, consulté le 24 avril 2019. URL : http://journals.openedition.org/rdlc/2172 ; DOI : $10.4000 /$ rdlc. 2172

Ce document a été généré automatiquement le 24 avril 2019

\section{$\Theta \Theta \Theta \Theta$}

Recherches en didactique des langues et des cultures is licensed under a Creative Commons AttributionNonCommercial-NoDerivatives 4.0 International License 


\title{
La photographie, déclencheur d'interaction en classe de langue : négociations entre apprenants
}

\author{
Catherine Muller
}

\section{Introduction}

\subsection{Objet de recherche et méthodologie}

1 La didactique des langues étrangères, dans ses dimensions méthodologiques, a entretenu et entretient encore un rapport étroit avec les images. En effet, des supports visuels sont utilisés en cours de langue pour activer la réception écrite et orale, ainsi que la production écrite et orale. Notre recherche porte sur l'utilisation de la photographie d'art en classe de langue pour susciter la parole et l'interaction entre les apprenants. Pour cela, une observation participante a été menée dans des classes de français enseigné comme langue étrangère à Paris, afin de recueillir un corpus de séquences de classe dans lequel les apprenants commentent des photographies d'art. Nous avons sélectionné pour cet article la photographie de D. Issermann Honor Frazer Yohjiyamoto ${ }^{1}$. Elle a été montrée à 4 groupes de différents niveaux de compétence : SEQ 1 (niveau A1), 2 (niveau A1), 3 (niveau B1), 4 (niveau B2). Ces apprenants sont originaires de différentes aires culturelles: Amérique latine, Moyen-Orient, Asie et Europe de l'Est.

\subsection{Cadres théoriques}

2 L'analyse des interactions à partir des photographies nécessite l'articulation de différents champs disciplinaires. Cette recherche est en continuité avec la réflexion sur les méthodologies d'enseignement en didactique des langues. L'utilisation de la photographie soulève en effet la question des supports d'enseignement. L'image a joué un rôle central dans le courant structuro-global audio-visuel (SGAV), non seulement pour traduire visuellement des énoncés, mais également dans le cadre de la production sur 
images. Cependant, les manuels SGAV ont provoqué des réactions de rejet en raison de leurs images univoques et schématiques et de leur caractère répétitif. Depuis, des activités originales à partir de photographies ont été proposées par C. de Margerie (1979) et $\mathrm{F}$. Yaiche (2002).

Cette étude rejoint également une problématique d'enseignement classique, à savoir le traitement de l'interaction orale: comment faire parler les apprenants? Comment susciter des interactions en classe de langue? Dans la méthodologie de l'approche par compétences (Beacco, 2007), sont proposés des échantillons qui constituent des modèles. L'activité mise en œuvre ici prend le contre-pied de cette démarche méthodologique puisqu'elle consiste à ne pas fournir d'input discursif : l'enseignante montre seulement une photo dont la force déclenche une verbalisation.

4 La forme d'interaction déclenchée par l'observation des photographies sera caractérisée à l'aide des outils de l'"analyse $d u$ discours en interaction" développée par C. Kerbrat-Orecchioni (2005). On s'appuiera également sur les analyses propres aux interactions en classe de langue. La bifocalisation de l'attention a été mise en évidence par P. Bange (1992): la classe de langue est tantôt focalisée sur le code (dimension métalinguistique) et tantôt sur le contenu (dimension mondaine).

D'autres champs disciplinaires, tels que les recherches sur la réception des œuvres d'art, l'anthropologie des images et l'identité culturelle permettront de mieux comprendre ce qui se joue dans l'activité. '

\subsection{Questions de recherche}

6 L'analyse du corpus porte sur l'influence d'un document photographique sur la structure et les contenus des interactions. L'étude s'attachera à analyser le format de la séquence et à comprendre ce qui est en jeu dans cette activité de classe atypique (Bigot \& Cicurel, $2005: 5)$. On s'interrogera aussi sur les perspectives didactiques concernant l'utilisation de la photographie comme support d'enseignement. '

\section{Une situation propice à la négociation}

7 Tout d'abord, l'interaction déclenchée est propice à la négociation. Les apprenants interagissent dans un mode de communication proche de la conversation et prennent position.

\subsection{Expression libre semi-guidée}

8 La forme d'interaction déclenchée présente une originalité forte. Elle s'éloigne de L'enseignante ne cherche pas à enseigner une signification des photographies. Elle ne fournit aucune indication sur le titre, la légende ou le nom du photographe. L'interaction favorise la communication directe entre apprenants qui ont des interventions initiatives. L'analyse de la distribution de la parole met en évidence la présence de prises de parole auto-sélectionnées. L'exemple suivant témoigne d'une construction collective sans intervention de l'enseignante : 


\begin{tabular}{|l|l|l|}
\hline SEQ 4: & & \\
\hline 157 & Serghei & peut-être elle est très très belle et cachée \\
\hline 158 & Am & elle est tombée dans cette \\
\hline 159 & Serghei & et puis elle essaie de : \\
\hline 160 & Luiza & sortir \\
\hline 161 & Serghei & de sortir \\
\hline 162 & Am & c'est pas possible \\
\hline 163 & Samir & elle est coincée \\
\hline
\end{tabular}

9 Ces échanges sont marqués par une communication entre apprenants. On observe une progression dans l'échange : la femme est très belle et cachée, elle est tombée et elle essaie de sortir, mais ce n'est pas possible car elle est coincée. Ce scénario est construit par plusieurs apprenants.

10 Cette répartition spécifique de la parole a été mise en évidence par différents auteurs dans certains corpus. V. Bigot note, dans le corpus qu'elle analyse, la présence de longs passages sans intervention de l'enseignant: "les tours de parole sont auto-sélectionnés et non pas attribués par le professeur, comme c'est souvent le cas dans un cours de langue" (Bigot, 1996 : 35). F. Carraud souligne que le débat en classe se caractérise par "un autre mode de circulation de la parole: les élèves n'ont pas à répondre au maître, ils s'adressent à leurs pairs et peuvent avoir des interventions initiatives" (Carraud, 2005 : 76). Ces remarques s'appliquent également au corpus analysé.

\subsection{Absence de consensus}

11 On observe une absence de consensus. L'activité incite les apprenants à échanger leurs points de vue sur la photographie. Cela conduit à la mise en place de débat :

\begin{tabular}{|l|l|l|}
\hline SEQ 2 : & & \\
\hline 017 & Nasiba & la montagne y a est fermée $\uparrow$ \\
\hline 023 & Nasiba & la femme \\
\hline 024 & Mircea & montagne fermée c'est pas possible \\
\hline 025 & Nasiba & elle ne veut pas fermer (rires) \\
\hline 029 & Mircea & $\begin{array}{l}\text { montagne \#fermée\# ça n'existe pas } \\
\text { montagne fermée c'est pas vrai }\end{array}$ \\
\hline
\end{tabular}


Dans cet exemple, on observe un désaccord entre les apprenants. Nasiba suggère que la montagne est fermée et que la femme lutte contre cette fermeture. Mircea réfute cette idée avec l'argument "ça n'existe pas montagne fermée c'est pas vrai". Selon A. Baptiste et al., une méthode comme Photolangage qui encourage la communication en groupe à partir de photographies est "peu adaptée à la recherche d'un consensus sur un thème donné" (Baptiste et al., $1991: 23)$.

\subsection{La notion de négociation}

On observe ainsi des divergences entre les apprenants concernant la perception et l'interprétation de la photographie. Ces désaccords donnent lieu à une négociation conversationnelle. La négociation, telle que définie par C. Kerbrat-Orecchioni (2005), implique un désaccord que l'on tente de résoudre par la coopération. Elle constitue un processus qui vise à résoudre un différend. Ce concept semble particulièrement pertinent dans la situation analysée. A. Trognon souligne que "le processus de négociation des assertions constitue certainement une propriété importante de l'activité pédagogique" (Trognon, 1993 : 118). Différentes issues sont possibles. Cette négociation peut mener à une solution négociée avec ajustements (Traverso, $2004: 146)$, ou bien un locuteur peut adhérer à la position d'un autre. Dans d'autres cas, les positions sont maintenues et la négociation n'aboutit pas.

\subsection{Différents types de négociation}

C. Kerbrat-Orecchioni (2004: 30) évoque les composantes du discours sur lesquelles peut porter la négociation. Il s'agit (1) de dimensions formelles, comme le registre ou la langue, (2) d'aspects organisationnels, comme la structuration des échanges et les tours de parole, (3) du contenu de l'échange, par exemple l'expression de l'opinion, (4) des identités des interactants et de la relation interpersonnelle.

Dans notre corpus, les désaccords sont de différentes natures. La négociation porte sur trois composantes principales, que nous développerons. Tout d'abord, la négociation métalinguistique porte sur les signifiants et leur référent, la dénomination et l'interprétation des mots. Elle concerne davantage la description. Ensuite, la négociation du contenu porte sur le sens de la photographie. Elle concerne principalement l'interprétation. Enfin, on observe une négociation des identités culturelles.

\section{Négociation métalinguistique}

La négociation peut concerner les signes et leurs référents (Traverso, 2004: 146). L'activité de commentaire de photographie ("Qu'est-ce que vous voyez?") entraîne les apprenants à décrire les images. Or l'activité de description est d'ordre métalinguistique. Elle établit en effet une équivalence entre une image et un signifiant, qui est propre à la dénomination. 


\section{1. "Panne lexicale"}

17 On observe des négociations métalinguistiques lorsqu'un apprenant vient en aide à un autre en apportant le mot qui lui manque, à l'occasion d'une "panne lexicale" (Traverso, 1999 : 79). Les propositions de lexique sont interprétées comme justes ou fausses, elles sont ratifiées ou non. Dans l'extrait suivant, la demande métalinguistique n'est pas explicite :

\begin{tabular}{|l|l|l|}
\hline SEQ 3 : & & \\
\hline 041 & Djibril & $\begin{array}{l}\text { nous on part mais la vie reste comme des : } \\
\text { des : des : des : des : }\end{array}$ \\
\hline 042 & Roshan & cailloux \\
\hline 043 & Djibril & montagnes oui c'est \\
\hline
\end{tabular}

18 Au tour de parole 041, Djibril bloque sur le terme : il répète "des: des: des: des: des:". Roshan cherche à lui venir en aide en lui proposant le mot manquant "cailloux". Or, la proposition de Roshan ne correspond pas au terme que Djibril cherche à employer. Djibril n'accepte pas la dénomination formulée. J. Barthomeuf évoque la négociation du sens et de la forme chez les apprenants. Quand les apprenants se corrigent, "l'un des interlocuteurs se met en position d'apprenant et l'autre assume le rôle de l'expert" (Barthomeuf, 1991: 253). L'auteur note à propos de l'absence de ratification: "Lors des moments de négociation $d u$ sens, de même que la compétence de l'expert du moment est relative, son statut est également relatif et on ne lui accorde pas une confiance absolue" (ibid. : 257).

\subsection{Débat sur la dénomination}

La négociation peut provenir aussi d'un désaccord sur les signifiants lors de la dénomination des éléments de la photo par les apprenants. R. Jakobson (1963) distingue la fonction métalinguistique de la fonction référentielle. Certains désaccords semblent relever davantage de la fonction référentielle. Les apprenants sont en désaccord sur l'identité des référents de la photographie. A. Reich analyse "les efforts de coopération et de négociation entrepris par des interlocuteurs lorsqu'ils doivent se mettre d'accord sur l'identité d'un référent" (Reich, 1991: 122). On observe un débat sur la dénomination dans l'exemple suivant:

\begin{tabular}{|l|l|l|}
\hline SEQ 4: & & \\
\hline 064 & Abdel & noire et euh une chaussure blanc + euh blanches \\
\hline 065 & P & mmh \\
\hline 066 & Maruh & elle + elle n'a pas de chaussures c'est les pieds (rires) + je crois \\
\hline
\end{tabular}


Les apprenants désignent des éléments de la photo. L'échange est caractérisé par une négation puis une affirmation: "c'est pas X, c'est $Y$ ". Le présentatif "c'est" est marqueur d'équivalence. Maruh réfute la proposition d'Abdel pour suggérer sa propre dénomination. La modalisation avec l'expression "je crois" montre qu'elle insiste sur son interprétation personnelle de l'image.

Le débat entre les apprenants porte aussi sur les termes employés. Cette négociation est de nature métalinguistique quand les apprenants débattent du mot adéquat pour décrire l'image, comme dans l'exemple suivant :

\begin{tabular}{|l|l|l|}
\hline SEQ 4: & & \\
\hline 396 & Viktor & je peux dire que : : c'est quelque chose mythique \\
\hline 397 & P & quelque chose de mythique $\uparrow$ \\
\hline 398 & Viktor & oui \\
\hline 399 & Af & mystique \\
\hline 400 & P & ouais $\uparrow$ \\
\hline 401 & Viktor & non c'est pas mystique \\
\hline 402 & P & mythique $\uparrow$ \\
\hline 403 & Viktor & mythique \\
\hline
\end{tabular}

Une apprenante réagit à la proposition de Viktor qui emploie l'adjectif "mythique". Elle suggère l'adjectif "mystique" qui est refusé par Viktor. Il ne ratifie pas ce mot. Il y a un désaccord sur la signification et l'interprétation du mot.

\subsection{Construction collective du sens de l'image}

Les désaccords entre les apprenants et les efforts pour parvenir à un accord par la négociation permettent d'aboutir à une construction collective du sens de l'image.

\begin{tabular}{|l|l|l|}
\hline SEQ 1: & & \\
\hline 049 & Af & une robe \\
\hline 050 & Nurhan & noire \\
\hline 051 & Am & noire \\
\hline 052 & Nurhan & une robe noire \\
\hline 053 & Cesar & $\begin{array}{l}\text { je ne sais pas si c'est noir parce que + la } \\
\text { photocopie euh noire et blanche }\end{array}$ \\
\hline
\end{tabular}


L'exemple suivant présente un désaccord qui permet une émulation. Entre les tours de parole (TP) 049 et 052, on observe une construction collective : l'expression "une robe noire " est construite grâce à la collaboration entre plusieurs apprenants. La précision de Nurhan (avec l'emploi de l'adjectif "noir") entraîne Cesar à réfléchir aux couleurs réelles de l'image ("je ne sais pas si c'est noir parce que + la photocopie euh noire et blanche"), il réfute ainsi la proposition "une robe noire". Les autres apprenants ne le contredisent pas. Nurhan rallie même sa position en proposant d'autres couleurs : "rouge euh bleu marine marron".

\subsection{Négociation du sens et explications entre pairs}

Les apprenants ont parfois recours à des explications. Ces explications entre pairs semblent stimuler les apprenants, comme dans l'extrait suivant :

\begin{tabular}{|l|l|l|}
\hline SEQ 2: & & \\
\hline 171 & Wen & $\begin{array}{l}\text { et je sou- souvenir s- souvenir le euh tu } \\
\text { euh : vous sais le le [spadak] euh + 300 sol- } \\
\text { soldats [spadak] }\end{array}$ \\
\hline 180 & Wen & [spadak] \\
\hline 181 & Af & [spartak] \\
\hline 182 & Wen & 300 [spardak] \\
\hline 183 & Felipe & $*$ Esparta* \\
\hline 184 & Wen & 300300300 [spadak] sol- \\
\hline 185 & Felipe & AH \\
\hline 186 & Wen & soldats 300 soldats \\
\hline 193 & elipe & F 300 le film euh et : de $:{ }^{*}$ Esparta* \\
\hline 197 & Felipe & euh Léonidas oui $\uparrow$ \\
\hline
\end{tabular}

Wen met en relation la photo avec le film 300 qui relate la bataille entre les Spartiates et les Perses. Il essaie de prononcer Sparte "[spadak]". Une fois que Felipe a compris de quel film Wen parle, il négocie une traduction en employant sa langue, l'espagnol. Il traduit " Sparte" par "Esparta". Les apprenants négocient la traduction du film. Afin d'aider la compréhension, Felipe cite également le nom d'un personnage du film : "Léonidas".

Les photographies sont perçues différemment selon les spectateurs. On n'observe donc pas de consensus sur la description et l'interprétation. 


\section{Négociation du contenu}

\begin{tabular}{|l|l|l|}
\hline SEQ 4 : & & \\
\hline 109 & Luiza & elle va jamais y arriver \\
\hline 110 & Abdel & si si elle va y arriver \\
\hline 111 & Luiza & non elle va pas arriver + c'est un rocher il est XX \\
\hline
\end{tabular}

\subsection{Désaccords sur l'interprétation}

On observe des désaccords sur la signification de la photographie.

Cette activité pédagogique conduit aussi les apprenants à confronter leurs points de vue sur la photographie et son interprétation. Selon V. Traverso (2004: 146), la négociation peut porter sur les contenus et particulièrement les opinions exprimées par les interlocuteurs. Ch. de Margerie souligne que dans l'une de ses expériences, "un débat s'est aussitôt enclenché entre partisans de l'une ou de l'autre lecture" (Margerie, 2006 : 103).

L'échange suivant confronte des interprétations différentes de l'image. Il s'agit d'un désaccord sur l'issue de la photographie. Luiza suggère que la femme ne va pas arriver à sortir, ou à pousser le rocher. Cette proposition est niée par Abdel. Luiza reste sur sa position. Le débat est marqué par les réfutations avec l'emploi de la négation : "non". La réfutation d'Abdel entraîne Luiza à justifier sa position ("c'est un rocher"). Le désaccord est maintenu. La confrontation de points de vue différents amène ainsi les apprenants à justifier leur opinion par des arguments.

\subsection{Construction collective de l'interprétation}

Les interactions révèlent une construction collective de l'interprétation de l'image par le groupe. On observe une négociation entre les apprenants quant à la signification des photographies. Ils formulent des hypothèses auxquelles les autres membres du groupe réagissent par l'approbation ou le désaccord. Les apprenants peuvent adhérer aux propositions émises. Ils répètent des termes employés par leurs pairs ou bien complètent un énoncé amorcé par l'un d'entre eux. C'est le cas dans les séquences suivantes :

\begin{tabular}{|l|l|l|}
\hline SEQ 3 : & & \\
\hline 180 & Djibril & rencontre ça + mais $x++$ le caillou jamais va rencontrer \\
\hline 181 & Roshan & caillou rester comme ça \\
\hline 182 & Djibril & il va rester comme ça \\
\hline
\end{tabular}




Dans l'échange suivant, Salma complète l'amorce d'én
syllabe :
\begin{tabular}{|l|l|l|l|}
\hline SEQ 1: & & énoncé en suspens avec une intonation mon \\
\hline 283 & Diego & $\begin{array}{l}\text { parce que le visage c'est la représentation } \\
\text { de l'homme de la femme que : qui pousse } \\
\text { quelque chose pour : } \uparrow\end{array}$ \\
\hline 284 & Salma & la liberté (petit rire) \\
\hline
\end{tabular}
validée par Djibril qui répète l'expression "rester comme ça".

\section{3. Émulation}

Dans la séquence 3, Roshan paraphrase, reformule l'idée de Djibril. Sa proposition est

Dans l'échange suivant, Salma complète l'amorce d'énoncé de Diego. Ce dernier a en effet

Dans leurs différentes hypothèses, les apprenants se contredisent, ce qui crée une situation propice à l'émulation, comme dans l'exemple suivant :

\begin{tabular}{|l|l|l|}
\hline SEQ 4 : & \\
\hline 181 & Mehdi & elle fait du sport peut-être \\
\hline 182 & P & ah peut-être OUI (rires) \\
\hline 183 & Am & avec une si belle robe \\
\hline 186 & Serghei & $\begin{array}{l}\text { oui mais robe ça + on peut pas faire la } \\
\text { gymnastique hein + c'est une robe là + non } \uparrow\end{array}$ \\
\hline 188 & Af & XXX elle essaie XX \\
\hline 189 & Samir & $\begin{array}{l}\text { peut-être le matin parce qu'il y a la } \\
\text { lumière }\end{array}$ \\
\hline 191 & Samir & elle fait gymnastique le matin parce qu'il y a de la lumière c'est très bien \\
\hline
\end{tabular}

Cet extrait met en évidence un désaccord sur l'activité de la femme. Mehdi suggère que la femme fait du sport. Certains apprenants remettent en cause la possibilité d'une pratique sportive. Un apprenant réagit à la proposition de Mehdi, il réfute cette idée en donnant l'argument de la robe : "une si belle robe". Ce tour de parole fait réagir Serghei qui prend position et le justifie : "on peut pas faire la gymnastique hein + c'est une robe là + non $\uparrow$ ". Cette idée est elle aussi contestée. Samir fournit un contre-argument: "elle fait gymnastique le matin parce qu'il y a de la lumière c'est très bien". Il s'agit ici d'une émulation car les désaccords permettent aux apprenants de se justifier. Comme les divergences portent sur l'opinion des interlocuteurs, on aboutit à une situation argumentative (Plantin, 1996). 
Ainsi, les variations dans la perception des photos contribuent au débat entre les apprenants. Mais ces divergences amènent surtout une émulation par la négociation du sens dans l'interprétation des photographies.

La discussion porte sur l'interprétation d'une photographie, qui suscite des réactions culturelles non convergentes. En anthropologie, S. Conord souligne que "l'expression des différentes interprétations d'un cliché révèle la diversité des points de vue produits par la variété des trajectoires sociales et personnelles relatives à chaque individu" (Conord, 2007 : 19). Ce qui est en jeu est donc l'identité (culturelle) des apprenants.

\section{Négociation des identités culturelles}

Enfin, la négociation concerne les identités culturelles des apprenants. Les identités sont considérées ici comme étant le produit des interactions. Dans les commentaires de photos, on observe une présentation de soi directe ou indirecte. Selon E. Goffman "l'acteur doit agir de façon à donner, intentionnellement ou non, une expression de lui-même, et les autres à leur tour doivent en tirer une certaine impression" (Goffman, 1973 : 12).

\subsection{Construction interactive de l'identité : le cas des plaisanteries}

Les apprenants négocient leur identité et celle des autres à travers des remarques humoristiques. Il existe une coopération explicite lors des face-à-face apprenantsapprenants. Un apprenant s'adresse à un autre apprenant. On observe alors l'émergence de la personne derrière l'apprenant, avec sa personnalité, sa vie et son humour. Dans les extraits suivants, la négociation porte sur l'identité de deux apprenants : les plaisanteries sont adressées directement à des membres du groupe :

\begin{tabular}{|l|l|l|}
\hline SEQ 1: & & \\
\hline 176 & Diego & parce que je : je aller à psy- psychologue $\uparrow$ \\
\hline 178 & Diego & $\begin{array}{l}\text { et elle m'a demandé beaucoup de photos et } \\
\text { me dit vous que regardez } \uparrow\end{array}$ \\
\hline 184 & P & et après elle t'a dit tu es : $\uparrow$ \\
\hline 186 & P & tu as telle personnalité $\uparrow$ \\
\hline 187 & Diego & ah oui elle m'a dit ça \\
\hline 190 & Mario & tu es un Pérou \\
\hline 191 & As & (rires) \\
\hline 193 & Mario & elle a dit tu es un Péruvien \\
\hline
\end{tabular}

Diego explique qu'il a vu une psychologue qui lui a demandé de parler de photographies afin de cerner sa personnalité. Mario se moque de lui et suggère que la psychologue lui a simplement dit "tu es un Péruvien". Il s'agit d'une plaisanterie qui porte sur l'identité de 
Diego : "A propose à B une identité pour B" (Kerbrat-Orecchioni, 2005 : 159). Diego ne ratifie ni conteste cette proposition.

Cependant, il se moque de Mario quelques tours de parole plus tard :

\begin{tabular}{|l|l|l|}
\hline SEQ 1: & & \\
\hline 205 & Diego & quoi c'est Mario \\
\hline 206 & P & c'est Mario $\uparrow$ \\
\hline 207 & As & (rires) \\
\hline 208 & P & pourquoi $\uparrow$ (rires) pourquoi c'est Mario $\uparrow$ \\
\hline 209 & Diego & la barbe \\
\hline 210 & P & parce qu'il a une barbe $\uparrow$ \\
\hline 211 & Diego & la femme de la barbe \\
\hline 212 & As & (rires) \\
\hline
\end{tabular}

En effet, il laisse entendre que la femme représentée ressemble à Mario. Il argumente avec la présence d'une barbe sur le visage de la femme. Or, Mario porte la barbe. Indirectement, il sous-entend que Mario ressemble à une femme, à une femme à barbe. Mario ne ratifie ni ne conteste cette proposition. Diego semble lui avoir rendu la monnaie de sa pièce.

Cela nous incite à dire avec E. Klett que "rire n'a pas de rapport avec un contenu mais avec un vécu partagé des interlocuteurs engagés dans l'interaction" (Klett et al., 1994: 44) ; le rire " marque une complicité entre les participants" (ibid. : 44). Ainsi, "rire ensemble renforce les liens entre les membres d'un groupe" (Foerster, $1984: 147)$.

\subsection{Mise en relation des photographies}

Les apprenants construisent l'interprétation en mettant en relation la photographie avec leurs univers culturels de référence. Il s'agit d'un dispositif interprétatif renvoyant à un ensemble d'images, de textes et à un imaginaire codifié. Des traces d'une identité culturelle apparaissent dans les références des spectateurs à des productions culturelles. On observe des manifestations de l'identité de Nurhan dans cet extrait :

\begin{tabular}{|l|l|l|}
\hline SEQ 1: & \\
\hline 313 & Nurhan & $\begin{array}{l}\text { peut-être euh Samson et Dalila ou : L- L- } \\
\text { Lei- Samson }\end{array}$ \\
\hline 314 & Afs & (rires) \\
\hline
\end{tabular}




\begin{tabular}{|l|l|l|}
\hline 315 & Nurhan & $\begin{array}{l}\text { non Samson et Dalila ou : Lei- Leila ou : } \\
\text { quelque chose } \underline{\text { euh }}\end{array}$ \\
\hline 326 & Nurhan & ou Roméo et Juliette \\
\hline
\end{tabular}

Elle évoque des références culturelles qui reposent sur un héritage littéraire. Elle se réfère dans un premier temps à Samson et Dalila. En effet, Samson est doté d'une force exceptionnelle, qui lui permettrait certainement de pousser ce rocher. Ensuite, elle cite la légende de Leila et Majnoun, où il est question d'une grande montagne qui se présente comme un obstacle. Ces deux couples partagent le fait d'être composés de deux clans opposés, ce qui l'amène à évoquer Roméo et Juliette.

Dans la séquence 2, Nasiba évoque Ali Baba avec l'expression "*sim sim* ouvrir" ("sésame ouvre-toi") :

\begin{tabular}{|l|l|l|}
\hline SEQ 2 : & & \\
\hline 034 & Nasiba & $\begin{array}{l}\text { tu dois dire trois *sim sim* ouvrir *sim } \\
\text { sim* fermer (rires) }\end{array}$ \\
\hline
\end{tabular}

\subsection{Mémoire visuelle}

Les univers culturels de référence renvoient à la notion de culture visuelle. L'observation d'une photographie éveille des images mentales, par l'intermédiaire de la mémoire visuelle. L'anthropologie des images (Belting, 2004) expose la façon dont les images évoquent d'autres images. La culture visuelle apparait dans les références des spectateurs à d'autres images. Selon $\mathrm{N}$. Heinich, "Chaque rencontre avec une œuvre est désormais nourrie de toutes les images d'autres cuvres avec lesquelles nous établissons, même sans le savoir, des comparaisons" (Heinich, 2001 : 63-64).

Ainsi, dans la séquence 2, la photographie rappelle à Wen le film 300 :

\begin{tabular}{|l|l|l|}
\hline SEQ 2: & & \\
\hline 473 & Wen & $\begin{array}{l}\text { (montre l'affiche du film 300 sur l'écran de } \\
\text { son ordinateur) oui le le film sp- } \uparrow \mathrm{sp} \text { - } \\
\text { [spadak] }\end{array}$ \\
\hline
\end{tabular}

Il justifie cela à la fin de la séquence en montrant l'affiche du film sur Internet. Cette séquence se déroule dans une salle de multimédia.

L'image fixe éveille ainsi des souvenirs d'images animées. C'est le cas également de Cesar qui évoque une publicité pour le chocolat Ferrero et pour le parfum Nina Ricci :

\begin{tabular}{|l|l|l|}
\hline SEQ 1: & & \\
\hline
\end{tabular}




\begin{tabular}{|l|l|l|}
\hline 091 & Cesar & $\begin{array}{l}\text { \#oui\# c'est une caverne et c'est un photo } \\
\text { pour un [parfym] }\end{array}$ \\
\hline 542 & Cesar & je me rappelle \\
\hline 543 & Olivia & ma copine \\
\hline 544 & Cesar & $\underline{\text { une publicité pour chocolat }}$ \\
\hline 545 & P & pour du chocolat $\uparrow$ \\
\hline 546 & Cesar & $\underline{\text { si parce que je XX }}$ \\
\hline 547 & Olivia & parfum chocolat \\
\hline 548 & Salma & $\underline{\text { (rires) }}$ \\
\hline 549 & Cesar & Ferrero Ferrero \\
\hline 554 & Salma & chocolat ou *perfume* (rires) \\
\hline 573 & Cesar & Nin- Nina Ricci \\
\hline 576 & Cesar & c'est $\uparrow$ une femme ${ }^{\text {qué }}{ }^{*}+$ monte $\uparrow$ \\
\hline 578 & Cesar & un $\uparrow$ un montagne \\
\hline 580 & Cesar & de $: \uparrow$ pommes \\
\hline
\end{tabular}

51 Ces références conduisent Olivia et Salma à se moquer de Cesar: "parfum chocolat", " chocolat ou *perfume*". Cela montre que les apprenants s'écoutent attentivement. En effet, au tour de parole 091, Cesar a évoqué un parfum et ce n'est qu'au tour de parole 544 qu'il évoque une publicité pour du chocolat. Cependant, Olivia a retenu la première proposition et voit une inconstance. Elle se moque également de lui en 543 en complétant son amorce d'énoncé et en suggérant que la femme de la photographie rappelle à Cesar sa copine.

Enfin, Olivia s'adresse directement à Cesar : "tu regardes beaucoup beaucoup télé" :

\begin{tabular}{|l|l|l|}
\hline SEQ 1: & & \\
\hline 569 & Olivia & (à Cesar) tu regardes \\
\hline 571 & Olivia & beaucoup beaucoup télé \\
\hline
\end{tabular}

Il s'agit ici encore d'une construction interactive de l'identité par l'intermédiaire de plaisanteries. 


\section{4. Échanges interculturels, partage de connaissances}

Les perceptions culturelles qui se manifestent donnent lieu à des échanges interculturels. Lorsque les univers culturels ne sont pas partagés par l'ensemble du groupe, qui compte diverses nationalités, les apprenants peuvent s'expliquer et être source de savoir pour toute la classe. Les photos favorisent ainsi un partage de connaissances. Une telle activité peut "susciter la curiosité et l'ouverture d'esprit des élèves" (Byram et al., 2002 : 39), ce qui entre dans le cadre des dimensions interculturelles.

Dans la séquence 4, Maruh met en relation la photographie avec un mythe des Philippines. Cette histoire n'est pas connue de l'ensemble du groupe, ce qui l'entraîne à raconter la légende et à justifier les rapprochements avec la photographie :

\begin{tabular}{|l|l|l|}
\hline SEQ 4: & & \\
\hline 023 & Maruh & $\begin{array}{l}\text { AH avant c'est comme l' + une histoire aux } \\
\text { Philippines y a une montagne }\end{array}$ \\
\hline 025 & Maruh & $\begin{array}{l}\text { y a un monsieur qui qui tire vers + } \\
\text { comment on dit ça va comme ça }\end{array}$ \\
\hline 027 & Maruh & $\begin{array}{l}\text { après ça a explosé les montagnes + y a un } \\
\text { monsieur qui + + il a + il a coupé euh le }: \\
\text { le }: \text { les montagnes et il a donc mis comme } \\
\text { ça + ça a bien + ça a sauvé le monde }\end{array}$ \\
\hline 029 & Maruh & ou $:$ là-bas $+y$ a l'histoire comme ça \\
\hline 030 & Mehdi & c'est une belle histoire \\
\hline
\end{tabular}

Les verbalisations devant des photographies d'art révèlent l'identité culturelle de spectateurs. Ces interactions favorisent ainsi des échanges culturels entre apprenants.

\subsection{La médiation culturelle}

Les apprenants sont amenés à gérer la diversité et à négocier des identités culturelles multiples. Il s'agit ainsi d'un lieu de médiation culturelle (Lévy \& Zarate, 2002).

\begin{tabular}{|l|l|l|}
\hline SEQ 2: & & \\
\hline 529 & Bachar & $\underline{\text { tu connais }}$ \\
\hline 530 & Mircea & $\underline{\text { Ali Baba et les } 40 \text { voleurs }}$ \\
\hline 531 & Bachar & $\begin{array}{l}\text { (à Wen }) \text { tu connais cette histoire en Chine } \\
\uparrow\end{array}$ \\
\hline 532 & Wen & $\underline{\text { Ali Baba }} \uparrow$ \\
\hline
\end{tabular}




\begin{tabular}{|l|l|l|}
\hline 533 & Bachar & Ali Baba \\
\hline 534 & Wen & oui euh \\
\hline 535 & As & (rires) \\
\hline 536 & Bachar & c'est mondial \\
\hline 537 & P & $\begin{array}{l}\text { oui en France aussi on connaît hein }+ \text { Ali } \\
\text { Baba }\end{array}$ \\
\hline 538 & Mircea & c'est comme Dracula en Roumanie \\
\hline
\end{tabular}

Dans l'exemple suivant, Bachar, un apprenant syrien, s'étonne que Wen, un apprenant chinois, connaisse l'histoire d'Ali Baba et les quarante voleurs : "tu connais cette histoire en Chine $\uparrow "$ ". Il en conclut que cette histoire est mondiale (TP 536). L'enseignante souligne que cette légende est aussi connue en France. Cela entraîne Mircea, un apprenant roumain, à comparer la célébrité d'Ali Baba avec la célébrité de la légende de son pays, à savoir Dracula. Il ne veut pas être en reste par rapport aux apprenants du Moyen-Orient. La référence à Dracula ne provient pas d'une ressemblance avec la photo. Les apprenants négocient ainsi leur héritage littéraire et culturel, que l'on pourrait reformuler ainsi : " vous avez Ali Baba, nous nous avons Dracula".

\subsection{Circulation entre les cultures}

On observe une circulation entre les cultures: les références des spectateurs viennent non seulement de leur culture d'origine, mais aussi de celles d'autres pays. Cela traduit ainsi les identités culturelles multiples des apprenants. Le Cadre européen commun de référence valorise la compétence pluriculturelle (Conseil de l'Europe, 2001: 105). M. Abdallah-Pretceille (1999) met en évidence l'hétérogénéité de plus en plus forte de la société. L'identité est plurielle et sa composition est labile. Un individu n'est pas nécessairement le représentant de sa culture. Il est possible de conjuguer son identité à sa manière, comme le montrent ces extraits de la séquence 2 :

\begin{tabular}{|l|l|l|}
\hline SEQ 2 : & & \\
\hline 029 & Mircea & $\begin{array}{l}\text { montagne \#fermée\# ça n'existe pas } \\
\text { montagne fermée c'est pas vrai }\end{array}$ \\
\hline 034 & Nasiba & $\begin{array}{l}\text { tu dois dire trois *sim sim* ouvrir * sim } \\
\text { sim* fermer (rires) }\end{array}$ \\
\hline 044 & Mircea & AH OUI je vois quelque chose \\
\hline 501 & Mircea & $\frac{\text { sim sim }^{*}}{}$ \\
\hline 503 & Mircea & ça c'est dans \\
\hline 518 & Mircea & 40 voleurs \\
\hline
\end{tabular}


Nasiba évoque la légende d'Ali Baba en citant la formule "*sim sim* ouvrir", "sésame ouvretoi". Mircea, qui avait contesté la possibilité d'une montagne fermée, se rallie à la position de Nasiba et il est le premier dans la séquence à prononcer le nom d'Ali Baba (TP 520) alors que Nasiba a dit "*sim sim* ouvrir" dès le tour de parole 34. L'issue du désaccord entre Mircea et Nasiba est donc une solution négociée.

\section{Conclusion : perspectives didactiques concernant la négociation en classe de langue}

61 La négociation présente des intérêts didactiques. Dans l'activité analysée, les apprenants construisent collectivement le sens de la photographie et interprètent l'image de façon interactive. Cette situation didactique permet d'apprendre à interagir et à débattre dans la langue cible. Elle peut constituer un entraînement à gérer des situations de communication complexes, comme la conversation à bâtons rompus, où le cours de l'échange est ouvert et les participants peuvent changer de sujet.

62 L'interaction déclenchée par la photo favorise un apprentissage entre pairs. On peut parler ici avec F. Cicurel de "coopération" et de "co-apprentissage" (Cicurel, 1994: 100). La photographie fonde un échange de points de vue entre les apprenants, permettant aux perceptions culturelles de se manifester. L'activité à partir de photographies encourage les apprenants à gérer indirectement les relations interculturelles. Le partage de connaissances favorise la découverte d'autres univers culturels, ce qui est propice à l'éducation interculturelle.

Pour terminer, citons Serghei dans la séquence 4 :

\begin{tabular}{|l|l|l|}
\hline SEQ 4 : & & \\
\hline 274 & Serghei & $\begin{array}{l}\text { j'aimerais bien sur mon mur dans ma } \\
\text { chambre une photo comme ça }\end{array}$ \\
\hline 283 & Serghei & $\begin{array}{l}\text { peut-être tous les jours je trouve quelque } \\
\text { chose d'autre tous les jours quelque chose } \\
\text { nouveau sur cette photo }\end{array}$ \\
\hline
\end{tabular}

\section{BIBLIOGRAPHIE}


Abdallah-Pretceille, M. (1999). L'éducation interculturelle. Paris : PUF.

Bange, P. (1992). "À propos de la communication et de l'apprentissage de L2 (notamment dans ses formes institutionnelles)". Acquisition et Interaction en Langue Étrangère, $\mathrm{n}^{\circ}$ 1, pp. 53-85.

Baptiste, A., Belisle, C., Pechenart, J.-M. \& Vacheret, C. (1991). Photolangage. Une méthode pour communiquer en groupe par la photo. Paris : Les éditions d'organisation.

Barthomeuf, J. (1991). "Asymétrie et apprentissage dans les activités de groupe en classe". In Russier, C., Stoffel, H. \& Véronique, D. (dir.) Interactions en langue étrangère. Aix-en-Provence : Publications de l'université de Provence, pp. 249-258.

Beacco, J.-C. (2007). L'approche par compétences dans l'enseignement des langues. Paris : Didier.

Belting, H. (2001, trad. : 2004). Pour une anthropologie des images (traduit de l'allemand par Torrent, J.). Paris : Gallimard.

Bigot, V. (1996). "Converser en classe de langue : mythe ou réalité ?". In Cicurel, F. \& Blondel, E. (dir.) La construction interactive des discours de la classe de langue - Les Carnets du CEDISCO 4, Paris : Presses de la Sorbonne nouvelle, pp. 33- 46.

Byram, M., Gribkova, B. \& Starkey, H. (2002). Développer la dimension interculturelle de l'enseignement des langues. Une introduction pratique à l'usage des enseignants. Strasbourg : Division des politiques linguistiques, Conseil de l'Europe.

Carraud, F. (2005). "Des débats philosophiques en classe : parler ou ne pas parler ?" In Cicurel, F. \& Bigot, V. (dir.) Les interactions en classe de langue - Le français dans le monde, Recherches et applications, juillet 2005, pp. 75-86.

Cicurel, F. (1994). "Marques et traces de la position de l'autre dans les discours d'enseignement des langues". In Cicurel, F., Lebre \& M., Petiot, G. (dir.) Discours d'enseignements et discours médiatiques, pour une recherche de la didacticité - Les Carnets du Cediscor 2. Paris : Presses de la Sorbonne nouvelle, pp. 93-104.

Conord, S. (2007). "Usages et fonctions de la photographie", Ethnologie française : Arrêt sur images Photographie et anthropologie $\mathrm{n}^{\circ}$ XXXVII, 1, Janvier-Mars, pp. 11-22.

Conseil de l'Europe (2001). Un cadre européen commun de référence pour les langues : apprendre, enseigner, évaluer. Strasbourg : Conseil de l'Europe, Division des politiques linguistiques. Paris : Didier.

Foerster, C. (1984). "Le rire : aspect non verbal dans l'interaction". In Interactions : Les échanges langagiers en classe de langue. Grenoble : Ellug, Centre de didactique du français, pp. 139-148.

Goffman, E. (1973). La mise en scène de la vie quotidienne. Tome 1. La présentation de soi. Paris : Les éditions de Minuit.

Heinich, N. (2001). La sociologie de l'art, Paris : La découverte.

Jakobson, R. (1963). Essais de linguistique générale, tome 1. Paris : Les Ėditions de Minuit.

Kerbrat-Orecchioni, C. (2004). "Négociations conversationnelles". In Grosjean, M. \& Mondada, L. (dir.) La négociation au travail, Lyon : PUL.

Kerbrat-Orecchioni C. (2005). Le discours en interaction. Paris : Armand Colin.

Klett, E., Lucas, M., Scipioni, L. \& Vidal, M. (1994). "Pour une recherche des positions discursives : l'enseignant et l'apprenant en situation d'interview". In Cicurel, F., Lebre \& M., Petiot, G. (dir.) Discours d'enseignements et discours médiatiques, pour une recherche de la didacticité - Les Carnets du CEDISCOR 2, Paris : Presses de la Sorbonne nouvelle, pp. 35-48. 
Margerie (de), C. (1979). "L'image dans l'enseignement audio-visuel des langues" In Margerie (de), C. \& Porcher, L. (1981) Des media dans les cours de langues. Paris : Clé international, pp. 21-60. Margerie (de), C. (2006). Entretien personnel. In Muller, C. (2007) L'image comme déclencheur de l'expression. Analyse des interactions en classe face à des photographies, Annexes, mémoire de master 2 recherche, Université Paris 3, pp. 101-114.

Plantin, C. (1996). "Le trilogue argumentatif. Présentation de modèle, analyse de cas", Langue française, $\mathrm{n}^{\circ} 112$, pp. 9-30.

Reich, A. (1991). "Désignation et description d'objets. Stratégies de compensation". In Russier, C., Stoffel, H. \& Véronique, D. (dir.) Interactions en langue étrangère. Aix-en-Provence : Publications de l'université de Provence, pp. 119-129.

Traverso, V. (1999). L'analyse des conversations. Paris : Nathan université.

Traverso, V. (2004). "Grille d'analyse des discours interactifs oraux". In Beacco, J.-C., Bouquet, S. \& Porquier, R. (dir.) (2004) Niveau B2 pour le français (utilisateur / apprenant indépendant). Textes et références. Strasbourg : Division des Politiques linguistiques, Paris : Didier, pp. 119-148.

Trognon, A. (1993). "La négociation du sens dans l'interaction". In Halté, J-F. (dir.) Inter-actions. L'interaction, actualités de la recherche et enjeux didactiques. Metz : Collection Didactique des textes. Centre d'Analyse Syntaxique de l'Université de Metz, pp. 91- 120.

Yaiche, F. (2002). Photos-Expressions. Paris : Hachette.

\section{ANNEXES}

Annexe 1 : conventions de transcription

\begin{tabular}{|l|l|}
\hline $\mathrm{XX}$ & segment inaudible \\
\hline (rires) & commentaire sur le non-verbal \\
\hline$:$ & allongement de la syllabe \\
\hline$:$ & allongement plus long de la syllabe \\
\hline+ & pause \\
\hline++ & pause plus longue \\
\hline$\uparrow$ & intonation montante \\
\hline- & mot tronqué \\
\hline AH OUI & accentuation, emphase intonative \\
\hline maintenant & chevauchement \\
\hline$* *$ & langue autre que langue-cible (que le français) \\
\hline$\#$ garçon\# & segment incertain (compréhension incertaine du segment) \\
\hline
\end{tabular}




\begin{tabular}{|l|l|}
\hline [ ] & transcription en alphabet phonétique international \\
\hline P & professeur \\
\hline Cesar & prénom de l'apprenant quand identifié \\
\hline Af & non identifié (femme) \\
\hline Am & non identifié (homme) \\
\hline As & plusieurs apprenants \\
\hline Afs & plusieurs apprenantes \\
\hline $\begin{array}{l}\text { Les prénoms des apprenants ont été modifiés en tenant compte de leur sexe et de leur } \\
\text { nationalité. }\end{array}$
\end{tabular}

\section{Annexe 2}

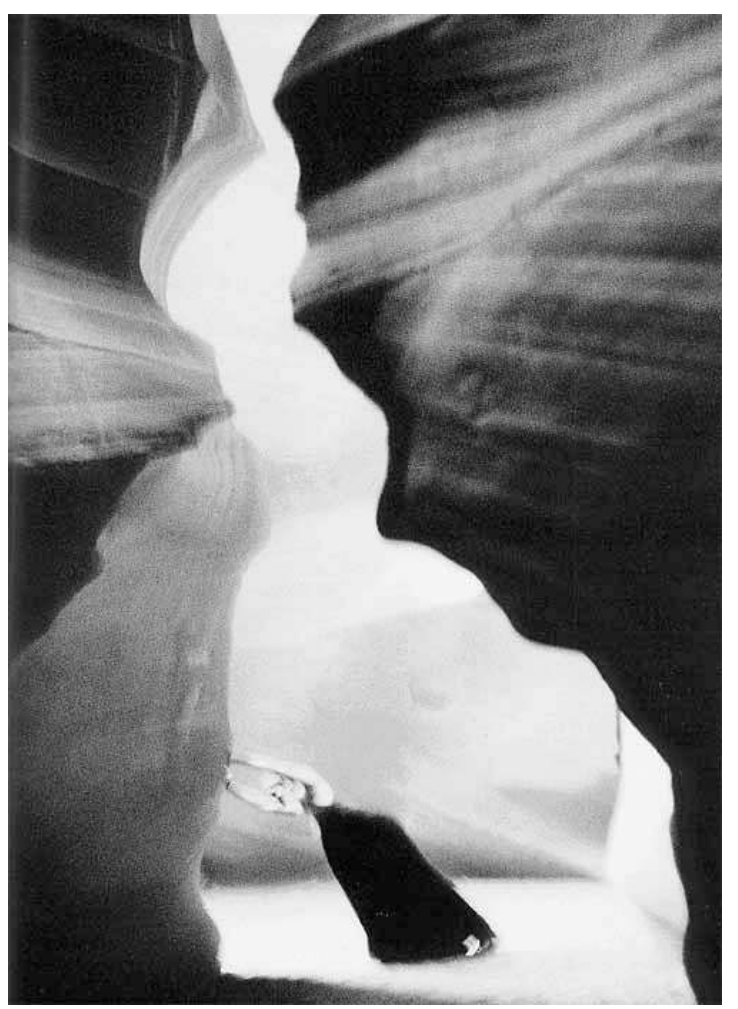

D. ISSERMANN Honor Frazer Yohjiyamoto, Arizona, 1996

\section{NOTES}

1. Voir annexe 2. 


\section{RÉSUMÉS}

Dans le cadre d'une observation participante, des photographies d'art ont été utilisées comme support didactique afin de déclencher la parole en classe de français enseigné comme langue étrangère. L'analyse de ces séquences de classe porte sur la forme et les enjeux des interactions, ainsi que sur les perspectives didactiques apportées par une telle activité. Le corpus mettant en évidence des divergences entre les apprenants, la notion de négociation permet de rendre compte des efforts des participants pour trouver un accord.

Cette négociation est tout d'abord de nature métalinguistique. Le débat entre les apprenants porte sur les signes et leurs référents. Cette activité pédagogique conduit aussi les apprenants à confronter leurs points de vue sur les significations de la photographie. La négociation du contenu débouche sur une construction collective du sens de l'image. Enfin, la négociation concerne les identités culturelles des apprenants : ces derniers mettent les photographies en relation avec leurs univers culturels. Cette situation didactique constitue un lieu d'échanges interculturels et favorise un apprentissage entre pairs.

This contribution deals with participant observation of students learning French as a foreign language. Artistic photographs were used to trigger oral expression. The analysis of the corpus examines the specificities of the interaction, and its relevance to foreign language teaching. The interaction highlights disagreements between students; thus the notion of "negotiation" can be used to account for the participants' efforts to find common ground.

Initial negotiation is metalinguistic. Students debate about signs and their references. The activity also drives students to confront their opinions on the interpretation of the photograph. The negotiation of content leads to a co-construction of the meaning of the picture. Finally, students negotiate their cultural identities. While commenting on the photograph, they refer to their cultural worlds. The activity thus encourages students to have intercultural exchanges and to learn from their peers.

\section{INDEX}

Mots-clés : interaction orale, négociation conversationnelle, dimension interculturelle, support didactique

Keywords : oral interaction, conversational negotiation, interculturality, course material

\section{AUTEUR}

\section{CATHERINE MULLER}

Catherine Muller est allocataire-monitrice à Paris 3 - Sorbonne Nouvelle. Elle prépare une thèse de doctorat en didactique des langues et des cultures au Diltec, sous la direction de Jean-Claude Beacco. 
Courriel : catherine.muller9[at]gmail.com

Adresse : Université Paris 3, UFR Dfle, 46, rue St Jacques 75005 Paris, France. 